\title{
Minor salivary glands in oral mucosa of two dogs: A pilot study
}

\author{
Gisha G. Nair ${ }^{1}$, S. Sooryadas ${ }^{2}$, C. V. Rajani ${ }^{3}$, P. T. Dinesh², \\ Harshad Patki Sudhir ${ }^{3}$, N. S. Jinesh Kumar ${ }^{2}$ and George Chandy ${ }^{2}$ \\ Department of Veterinary Surgery and Radiology, College of Veterinary and Animal Sciences, \\ Kerala Veterinary and Animal Sciences University, Pookode
}

Citation: Gisha, G.N., Sooryadas, S., Rajani,C.V., Harshad,P.S.,Dinesh, P.T., Jineshkumar,N.S. and George Chandy. 2021. Minor salivary glands in oral mucosa of two dogs: a pilot study. J.Vet. Anim. Sci. 52(1): 99-101. DOI: https://doi.org/10.51966/jvas.2021.52.1.99-101

Received: 29.09.2020

Accepted: 24.10 .2020

Published: 01.01.2021

\begin{abstract}
Presence of minor salivary glands in the oral mucosa and its potential use as an autograft for treating dry eye disease has been widely reported in humans. But there is a paucity of literature regarding the histological evidence for the presence of minor salivary glands in the oral mucosa of dogs. The present pilot study histologically identified and topographically described the minor salivary glands in the oral mucosa of two dogs, as a potential autograft for keratoconjunctivitis sicca. The samples were collected from different sites of the upper and lower lips and the commissures. Samples were processed for routine paraffin embedding; $5 \mu \mathrm{m}$ sections were taken and were stained using Hematoxyline and Eosin staining. Histological examination revealed exocrine, compound, tubulo-acinar glands and the secretion was mixed type.
\end{abstract}

Keywords: Minor salivary glands, canine, Keratoconjunctivitis sicca, $H$ and E staining

Salivary glands are mainly classified into minor and major salivary glands. The parotid, mandibular, monostomatic and polystomatic sublingual, and zygomatic glands are categorised as major salivary glands, while the labial, buccal, molar, lingual, and palatine are grouped under the minor salivary glands in the canine species (Evans and de Lahunta, 2013; Gioso and Carvalho, 2005). The minor salivary glands may be mucous, serous or mixed, and are the miniaturized forms of the major salivary glands (Frappier, 2006). These glands can be located in close proximity to the epithelial lining and in the interior of the lamina propria-submucosa in dogs (Dyce et al., 2004; Frappier, 2006). Murube (1997) reported the existence of a large number of minor salivary glands between the quadratus labi and the lip or the buccal mucosa and had described labial minor salivary glands as a potential alternative lubricant source for the ocular surface in humans for dry eye disorder.

1. MVSc Scholar, Email: aamis2011@gmail.com, Phone number: 9645406822

2. Assistant Professor

3. Assistant Professor, Department of Veterinary Anatomy and Histology

Copyright: () 2021 Gisha et al. This is an open access article distributed under the terms of the Creative Commons Attribution 4.0 International License (http://creativecommons.org/licenses/by/4.0/), which permits unrestricted use, distribution, and reproduction in any medium, provided the original author and source are credited. 


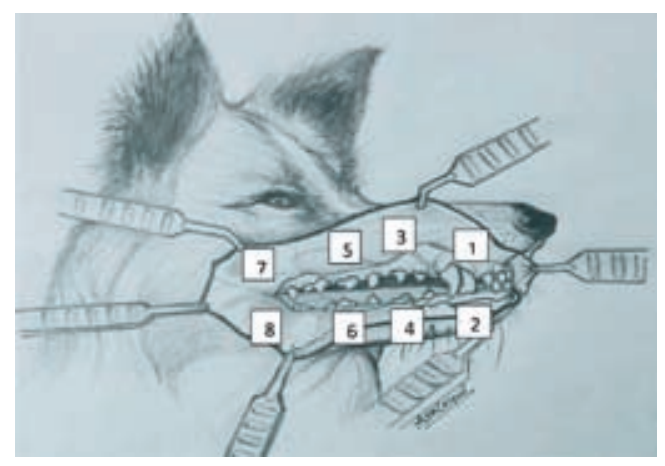

Fig.1 Sample collection sites within the oral cavity. Samples were collected from the upper rostral labial mucosa (1), lower rostral labial mucosa (2), upper labial mucosa from canine to third premolar (3)and (5), lower labial mucosa from canine to third premolar (4) and (6) and buccal mucosa near the commissure (7) and (8).

There are contradictions associated with the presence of minor salivary glands in dogs (Cherry et al., 2018). There is a paucity of literature regarding histological evidence for presence of minor salivary glands in oral mucosa of dogs. The present paper describes a pilot study on histological identification and topographical description of minor salivary glands in oral mucosa of two dogs.

Oral mucosal samples were collected from two adult dogs (one female Labrador aged 7 years and another Non-descript male aged 2 years) that had both died of reasons unrelated to the present study, for topographical identification of minor salivary glands in oral mucosa, as a potential autograft for dry eye disease in dogs. The samples were randomly collected from multiple sites (Fig. 1) of labial muco-cutaneous junction, labial mucosa of the upper and lower lips, and buccal mucosa. At every location, samples were collected from mucosa to the depth of muscularis. The collected samples were fixed in 10 per cent neutral buffered formaldehyde for 48 hours, in ambient temperature and processed using conventional histological techniques to embed in paraffin. Sections of $5 \mu \mathrm{m}$ thickness were taken and stained with Haematoxylin-Eosin for histological evaluation.

Sections from labial and buccal mucosa revealed mucosal, submucosal and the muscularis layers. Mucosa was lined by non-keratinised stratified squamous epithelium,

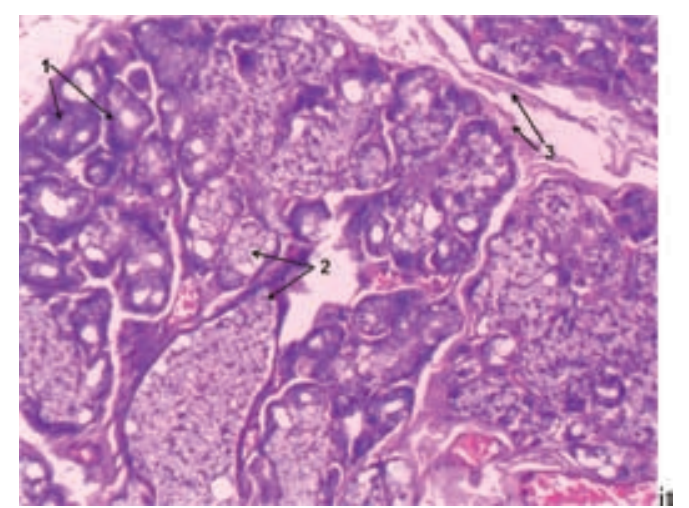

Fig. 2 Photomicrograph of oral mucosal sample from canine to third premolar in dogs showing minor salivary glands. $\mathrm{H}$ and $\mathrm{E} \times 150$. (1) Serous glands, (2) Mucus glands, (3). Connective tissue septa

while submucosa was composed of well vascularised dense irregular connective tissue. Small minor salivary glands were observed in sections of oral mucosa collected from the level of canine to third premolar. The glands were well encapsulated by connective tissue. The septa from the capsule divided the gland into lobules which enclosed secretory acini. Both serous and mucous secretory acini were present (Fig. 2). This indicated that the oral mucosa from the level of canine to third premolar contained plenty of mixed minor salivary glands. The glands were observed in the submucosal layer very close to the epithelium. The section of the oral mucosa from the other sites did not reveal any type of glands.

The present pilot study revealed that minor salivary glands were present in the oral mucosa at the level of canine to third premolar area and is in accordance with that reported by Nickel et al. (1979) who reported that minor salivary glands in dogs are localised in the area demarcated from canine to third cheek tooth. The minor salivary glands in the present study were observed close to the mucosal lining and a few were present close to the submucosa and were surrounded by connective tissue. These glands were mixed in nature.

Minor oral salivary glands account for half the baseline secretion of saliva and they exist in large numbers in the labial, buccal, and palatal mucosa in humans. Murube (1997) described transplantation of labial salivary 
glands for the treatment of severe dry eyes in humans. Labial salivary glands along with the overlying mucosa as an auto-graft have been transplanted to the eyelids to improve the ocular lubrication and to reduce discomfort in patients with kerato-conjunctivitis sicca (Marinho et al., 2010). Occurrences of minor salivary glands are reported in dogs. There are contradictions on the topography of glands and their existence in dogs. Cherry et al. (2018) reported that no minor salivary glands were observed in the canine oral mucosa after examining the oral biopsies of six randomly selected dogs.

The present pilot study in two dogs revealed that minor salivary glands were present in the area demarcated from canine to third premolar and were observed close to the mucosal lining. The presence of these minor salivary glands in oral mucosa potentiates its use as an auto-graft for treating dry eye disease in dogs. Further histological studies in a larger population and varied breeds of dogs are warranted.

\section{References}

Cherry, R. L., Smith, J. D. and Ben-Shlomo, G. 2018. Canine Oral Mucosa Evaluation as a Potential Autograft Tissue for the Treatment of Unresponsive Keratoconjunctivitis Sicca. Veterinary Ophthalmology 21(1):48-51

Dyce, K. M., Sack, W. O. and Wensing, C. J. G. 2004. Digestive Appartus. Treaty of
Veterinary Anatomy. $3^{\text {rd }}$ edn., pp. 99-145. Rio de Janeiro: Elsevier.

Evans, E. and de Lahunta, A. 2013. Miller's Anatomy of the Dog. $4^{\text {th }}$ edn., 299p, Philadelphia, PA: Elsevier.

Frappier, B. L. 2006. Digestive system. Dellmann's Textbook of Veterinary Histology. pp. 170-211, $6^{\text {th }}$ edn., lowa, Blackwell.

Gioso, M. A. and Carvalho, V. G. G. (2005). Oral Anatomy of the Dog and Cat in Veterinary Dentistry Practice. Veterinary Clinics of North America: Small Animal Practice. 35(4): 763-780.

Marinho, D.R., Burmann,T.Gand Kwitko, S.2010. Labial Salivary Gland Transplantation for Severe Dry Eye due to Chemical Burns and Stevens - Johnson syndrome. Ophthalmic Plastic and Reconstructive Surgery. 26(3):182-4.

Murube, J.1997. Substitute Surgery for Dry Eye and Glandular Transplants. Dry Eye. 1st edn., pp. 207-221, Tecnimedia Editorial, Madrid.

Nickel, R., Schummer, A., Seiferle, E. and Sack, W.O. 1979. The Viscera of the Domestic Animals. $2^{\text {nd }}$ edn., 401p, Berlin, Hamburg, Parey. 